Cahiers québécois de démographie

\title{
Les futurs linguistiques possibles de Montréal Aspects méthodologiques
}

\section{Mireille Baillargeon et Claire Benjamin}

Volume 6, numéro 3, décembre 1977

URI : https://id.erudit.org/iderudit/600746ar

DOI : https://doi.org/10.7202/600746ar

\section{Aller au sommaire du numéro}

Éditeur(s)

Association des démographes du Québec

ISSN

0380-1721 (imprimé)

1705-1495 (numérique)

Découvrir la revue

\section{Citer cet article}

Baillargeon, M. \& Benjamin, C. (1977). Les futurs linguistiques possibles de Montréal : aspects méthodologiques. Cahiers québécois de démographie, 6(3), 9-32. https://doi.org/10.7202/600746ar

\section{Résumé de l'article}

En plus de faire évoluer dans le temps de façon classique les populations de départ entre l'année $t$ et $t+1$, le modèle présente deux (2) caractéristiques importantes qui lui donnent son originalité et son intérêt.

1) Le modèle suit annuellement et simultanément par sexe et année d'âge, les populations de langue française, anglaise et autres de la région métropolitaine de Montreal, tant de langue maternelle (première langue apprise et encore comprise) que de langue d'usage (langue la plus souvent utilisée à la maison)

L'interdépendance de l'évolution de ces six (6) populations oblige à les suivre simultanément.

La croissance des populations selon la langue d'usage dépend de l'évolution de la croissance des populations selon la langue maternelle et de la tendance des transferts linguistiques (perte de la langue maternelle comme langue d'usage). Il faut donc, pour suivre dans le temps les populations selon la langue d'usage, suivre l'évolution des populations selon la langue maternelle. Or, ces dernières populations évoluent elles-mêmes en fonction de la croissance des populations selon la langue d'usage. En effet, les naissances, allant grossir par exemple les effectifs de la population de langue maternelle anglaise, sont le fait non seulement de mères de langue maternelle et d'usage anglaise, mais aussi de femmes de langue maternelle française ou autre et qui se sont anglicisées au point d'être devenues de langue d'usage anglaise. Par la natalité, les populations selon la langue maternelle sont donc, à leur tour, dépendantes de la croissance des populations selon la langue d'usage.

2) Le modèle permet de plus d'intégrer séparément les entrées et les sorties selon la langue maternelle, tant intraprovinciales qu'interprovinciales et internationales. Deux (2) raisons nous ont obligés à décomposer les migrations en ces différents niveaux. La première découle du fait que les migrants présentent des caractéristiques linguistiques bien différentes selon qu'ils viennent du reste du territoire québécois, des autres provinces canadiennes ou de l'étranger, ou au contraire, selon qu'ils partent de Montréal vers ces régions. La deuxième rejoint les préoccupations spécifiques du ministère de l'Immigration : pouvoir extraire l'effet d'une variation du volume ou de la composition des entrées internationales sur la population montréalaise.

En somme, l'intérêt du modèle, c'est son extrême souplesse. Il permet par le jeu de plusieurs simulations de mesurer les effets d'un changement d'un des facteurs de la croissance (mortalité, fécondité, mobilité géographique et mobilité linguistique) des ou d'une seule des populations linguistiques sur le volume, la structure par âge et la composition linguistique future de la population montréalaise.
Ce document est protégé par la loi sur le droit d'auteur. L’utilisation des services d'Érudit (y compris la reproduction) est assujettie à sa politique d'utilisation que vous pouvez consulter en ligne.

https://apropos.erudit.org/fr/usagers/politique-dutilisation/ 
Cahiers québecois de démographie

Vol. 6, no 3 spécial, décembre 1977.

BAILLARGEON Mireille et BENJAMIN Claire: Les futurs linguistiques possibles de Montréal: Aspects méthodologiques

\section{SOMMA IRE}

En plus de faire évoluer däns le temps de façon classique les po-

pulations de départ entre l'année $t$ et $t+1$, le modèle présente deux (2)

caractéristiques importantes qui lui donnent son originalité et son intéret.

1) Le modèle suit annuellement et simultanément par sexe et année d'âge, les populations de langue française, anglaise et autres de la région métropolitaine de Montréal, tant de langue maternelle (première langue apprise et encore comprise) que de langue d'usage (langue la plus souvent utilisée à la maison). L'interdépendance de l'évolution de ces six (6) populations oblige à les suivre simultanément.

La croissance des populations selon la langue d'usage dépend de l'évolution de la croissance des populations selon la langue maternelle et de la tendance des transferts linguistiques (perte de la langue maternelle comme langue d'usage). Il faut donc, pour suivre dans le temps les populations seIon la langue d'usage, suivre l'évolution des populations selon la langue maternelle. Or, ces dernières populations évoluent elles-mêmes en fonction de la croissance des populations selon la langue d'usage. En effet, les naissan- 
ces, allant grossir par exemple les effectifs de la population de langue maternelle anglaise, sont le fait non seulement de mères de langue maternelle et d'usage anglaise, mais aussi de femmes de langue maternelle française ou autre et qui se sont anglicisées au point d'être devenues de langue d'usage anglaise. Par la natalité, les populations selon la langue maternelle sont done, a leur tour, dépendantes de la croissance des populations selon la langue d'usage.

2) Le modèle permet de plus d'intégrer séparément les entrées et les sorties selon la langue maternelle, tant intraprovinciales qu'interprovinciales et internationales. Deux (2) raisons nous ont obligés à décomposer les migrations en ces différents niveaux. La première découle du fait que les migrants présentent des caractéristiques linguistiques bien différentes selon qu'ils viennent du reste du territoire québécois, des autres provinces canadiennes ou de l'étranger, ou au contraire, selon qu'ils partent de Montréal vers ces régions. La deuxième rejoint les préoccupations spécifiques du ministère de l'Immigration: pouvoir extraire l'effet d'une variation du volume ou de la composition des entrées internationales sur la population montréalaise.

En somme, l'intêrêt du modèle, c'est son extrême souplesse. Il permet par le jeu de plusieurs simulations de mesurer les effets d'un changement d'un des facteurs de la croissance (mortalité, fécondité, mobilité géo- 
graphique et mobilité linguistique) des ou d'une seule des populations linguistiques sur le volume, la structure par âge et la composition linguistique future de la population montréalaise. 
Cahiers cuébúcois de démographie

Vol. 6, no 3 spécial, Décembre 1977.

LES FUTURS LINGUISTIUUES POSSIBLES DE MONTREAL:

A SPECTS METHODOLOGIQUES

par

Mireille BAILLARGEON et Claire BENJAMIN *

1- LES DBJECTIFS DE L'ETUDE

Cette étude a deux (2) objectifs essentiels: premièrement, dé-

terminer le:s futurs linguistiques possibles de la région de Montréal ${ }^{1}$

en 2001 et deuxièmement, mesurer la sensibilité de chacune des variables

de la croissance des populations linguistiques sur ces évolutions possibles.

Cette étude est actuellement en cours de rédaction et devrait

se terminer vers le milieu de l'automne.

2- LE MODELE

Les objectifs de l'étude fixent les termes généraux du modéle que

* Direction de la Recherche, ministère de l'Immigration du juébec, 355, rue McGill, Montréal.

1 Région métropolitaine de Montréal selon les limites territoriales de 1971. 
nous avons construit ${ }^{l}$. En effet, pour atteindre avec une certaine précision ces deux (2) objectifs, il fallait élaborer un modèle qui puisse analyser et projeter séparément chaque composante de l'évolution démographique des populations francophone, anglophone et allophone ${ }^{2}$ de la région métropolitaine de Montréal.

La méthode des composantes répond à ces nécessités, puisqu'elle consiste à appliquer à une population de départ, une série de taux et de données correspondant à l'évolution prévue des facteurs d'évolution de cette population (mortalite, fécondite, migrations) pour obtenir la population projetée par sexe et âge de l'année suivante.

\subsection{Définitions des populations linquistiques}

En plus de la question sur la langue maternelle ${ }^{3}$ traditionnellement incluse par obligation légale dans les recensements canadiens, on a introduit pour la première fois dans le recensement de 1971 une question sur la langue d'usage ${ }^{4}$. Cette question a permis de constater que

1 Nous avons pu bénéficier des ccnseils et de l'expertise de Je\&ll MONGEAU démographe à l'I.N.R.S. - URBANISATION pour l'élaboration du modèle.

2 De langue autre que française et anglaise.

3 Première langue apprise et encore comprise.

4 Du usuelle: langue la plus souvent parlée à la maison. 
les populations rrancophone, anglophone et autre offraient une image différente de la situation linguistique à Montréal selon que l'on prenait comme critère d'appartenance a ces trois (3) groupes linguistiques, la langue maternelle ou la langue d'usage.

Les populations linguistiques selon la langue d'usage tiennent compte des changements linguistiques qui ont pu survenir dans la vie des individus entre leur naissance et l'année du recensement et décrivent mieux par conséquent la situation linguistique du moment que les populations selon la langue maternelle. Tout en étant un critère d'appartenance plus satisfaisant pour décrire une situation linguistique, nous savons bien que la langue d'usage elle-même ne décrit pas dans sa réalité totale cette situation puisque un grand nombre d'individus par exemple ne parlant ni le francais ni l'anglais à la maison doivent avoir opté pour l'une ou l'autre de ces deux (2) langues hors de leur domicile, que cela soit dans leur vie active ou scolaire, etc.

Malgré tout, l'utilisation de la langue d'usage, à défaut d'autres critères plus précis, ne manque pas d'intérêt. 


\subsection{Populations de départ et populations d'arrivée}

Le modele permet de suivre annuellement, par sexe et année d'âge, les populations francophone, anglophone et allophone, tant de langue maternelle que de langue d'usage.

En effet, si nous avons trois (3) populations de départ: les populations de langue maternelle française, anglaise et autre, nous avons annuellement six (6) populations d'arrivée: trois (3) selon la langue maternelle et trois ( 3 ) selon la langue d'usage.

\footnotetext{
L'interdépendance entre la croissance des populations linguistiques de langue maternelle et de langue d'usage nous oblige en effet à suivre annuellement ces deux (2) groupes de populations.
}

La croissance des populations selon la langue d'usage dépend de l'évolution des populations selon la langue maternelle et des tendances de la mobilité linguistique. Il faut done pour suivre dans le temps les populations de langue d'usage française, anglaise et autre, suivre aussi l'évolution des populations de langue maternelle française, anglaise et autre. Or, ces populations évoluent elles-mêmes en fonction de la 
croissance des populations selon la langue d'usage. En effet, les naissances allant grossir les effectifs de la population de langue maternelle française, par exemple, sont le fait de mères dont le français est la langue la plus souvent parlée à la maison, quelle que soit leur langue maternelle. Par la natalité, les populations selon la langue maternelle sont donc dépendantes de la croissance des populations selon la langue d'usage.

Pour simplifier quelque peu le modele, nous avons fait l'hypothèse que les populations de langue maternelle française et anglaise n'effectuent pas de transferts Iinguistiques ${ }^{1}$ vers une langue d'usage autre que l'anglais ou le français. Cette hypothèse néglige une force d'attraction aue les langues autres que le français ou l'anglais exercent auprès des communautés anglophone et francophone d'après le recensement de $1971^{2}$. Mais cette force est assez faible pour qu'on puisse la négliger, sans que cela modifie beaucoup nos résultats.

1 Perte de la langue maternelle comme langue d'usage.

2 En $1971,1,6 \%$ des personnes de langue maternelle anglaise et $0,3 \%$ des personnes de langue maternelle française utilisaient une langue autre que le francais et l'anglais de la maison. 


\subsection{Les variables du modèle}

Avant de présenter en détail les étapes nécessaires pour suivre

dans le temps les populations française, anglaise et autre de Montréal, tant de langue maternelle que de langue d'usage, nous avons rassemblé dans le tableau 1 toutes les variables et les symboles qui servent aux équations du modèle.

2.4 Evolution de la population de lancue maternelle française, anglaise et autre

La population de départ est la population de la région métropolitaine de Montréal selon le sexe, l'âge (par année d'âge) et la langue maternelle (française, anglaise et autre) au ler juin 1971.

A) Calcul des survivants de plus d'un (1) an

La population $P_{i}$ de langue maternelle $i$, d'âge $x-1$ au ler juin $t-1$, soit $x-1^{P} i(t-1)$, multipliée par la probabilité annuelle de survie (s) à l'âge $x-1$, selon la table de mortalité utilisée pour l'année $t-1$, donne la population survivante à l'âge $x$ de l'année suivante selon la formule:

$$
x_{i}^{P_{i}(t)=x-1} P_{i(t-1)} \cdot x-1{ }^{S}(t-1)
$$


Tableau 1 : Identification des variables du modèle

\begin{tabular}{|c|c|c|c|c|c|c|c|c|c|c|c|c|c|}
\hline \multirow{3}{*}{$\begin{array}{l}\text { POPULATIONS } \\
\text { DE DEPART } \\
\text { AU temps } \\
\text { t-1 } \\
\text { Populations } \\
\text { Belon la } \\
\text { langue ma- } \\
\text { ternelle }\end{array}$} & \multicolumn{2}{|c|}{$\begin{array}{l}\text { Accroissement } \\
\text { naturel }\end{array}$} & \multicolumn{6}{|c|}{$M O B I L I T E G E O G R A P H I Q U E$} & \multirow{3}{*}{\begin{tabular}{|l} 
POPULATIONS \\
D' ARRIVEE (1) \\
AU TEMPS t \\
$\begin{array}{l}\text { Populations } \\
\text { selon la } \\
\text { langue mater- } \\
\text { nelle }\end{array}$
\end{tabular}} & \multicolumn{3}{|c|}{ MOBILITE LINGUISTIQUE INTRA-GENERATION ** } & \multirow{3}{*}{\begin{tabular}{|l} 
POPULATIONS \\
D'ARRTVEE (2) \\
AU TEMPS $t$ \\
$\begin{array}{l}\text { Populations } \\
\text { selon la langue } \\
\text { d'usage }\end{array}$
\end{tabular}} \\
\hline & \multirow{2}{*}{$\begin{array}{l}\text { Probabilitié } \\
\text { de survie * }\end{array}$} & \multirow{2}{*}{$\begin{array}{l}\text { Taux de } \\
\text { fécon- } \\
\text { dité }\end{array}$} & \multicolumn{3}{|c|}{ IMMIGRATION } & \multicolumn{3}{|c|}{ EMIGRATION } & & \multirow{2}{*}{$\begin{array}{l}\text { Taux de conser- } \\
\text { vation de la } \\
\text { langue mater- } \\
\text { nelle }\end{array}$} & \multicolumn{2}{|c|}{ Taux de transfert } & \\
\hline & & & $\begin{array}{l}\text { Inter- } \\
\text { natio- } \\
\text { nale }\end{array}$ & $\left|\begin{array}{l}\text { Inter } \\
\text { provin- } \\
\text { ciale }\end{array}\right|$ & $\begin{array}{l}\text { Intra- } \\
\text { provin- } \\
\text { ciale }\end{array}$ & $\begin{array}{l}\text { Inter } \\
\text { natio- } \\
\text { nale }\end{array}$ & $\begin{array}{l}\text { Inter- } \\
\text { provin- } \\
\text { ciale }\end{array}$ & $\begin{array}{l}\text { Intra- } \\
\text { provin- } \\
\text { ciale }\end{array}$ & & & au français & $\grave{a} l^{\prime}$ anglais & \\
\hline${ }_{1}:$ française & $s$ & $v_{1}$ & $I_{1.1}$ & $I_{1.2}$ & $I_{1.3}$ & $E_{1.1}$ & $E_{1.2}$ & $E_{1.3}$ & $P_{1}:$ française & $c_{1}$ & - & $1-c_{1}$ & $P_{4}:$ françai \\
\hline${ }_{i}^{P}$ anglaise & $s$ & $v_{2}$ & $I_{2.1}$ & $I_{2.2}$ & $I_{\varepsilon .3}$ & $E_{2.1}$ & $E_{2.2}$ & $E_{2.3}$ & $P_{2}$ :anglaise & $c_{2}$ & $1-C_{2}$ & - & $P_{5}:$ anglaise \\
\hline$P_{3}:$ autre & $s$ & $v_{3}$ & $I_{3.1}$ & $I_{3.2}$ & $I_{3.3}$ & $E_{3.1}$ & $E_{3.2}$ & $E_{3.3}$ & $P_{3}:$ autre & $c_{3}$ & $c_{3.1}$ & $c_{3.2}$ & $P_{6}:$ autre \\
\hline
\end{tabular}

Hypozhèses : * Les groupes linguistiques sont soumis à la même table de mortalité.

** Les populations de langue maternelle française et anglaise sont supposées ne pas effectuer de transferts linguistiques vers une langue d'usage autre que l'anglais ou le français.

N.B. : $c_{3.1}+c_{3.2}=1-a_{3}$ 
Une même table de mortalité s'applique aux trois (3) groupes linguistiques par suite de notre décision de ne pas tenir compte de la mortalité différentielle.

B) Le solde migratoire pour les plus d'un (1) an Les migrants selon qu'ils viennent du reste de la province, des autres provinces canadiennes ou de l'étranger ou au contraire selon qu'ils partent vers ces trois (3) régions, représentent des caractéristiques linguistiques bien différentes. En effet, si la majorité des migrations entre Montréal et la province sont le fait de francophones, il n'en va pas de même pour les autres mouvements migratoires.

Pour tenir compte de ces différentes structures linguistiques des mouvements migratoires, nous avons construit un modele qui permet d'entrer des hypothèses sur le nombre total annuel des entrées et des sorties tant intraprovinciales, qu'interprovinciales et internationales par langue maternelle (française, anglaise, autre). Ces différents types de migrations sont répartis ensuite, selon une structure par âge et sexe qui leur est propre et que l'on suppose constante dans le temps, ce qui nous permet d'obtenir un solde migratoire total par âge et sexe pour 
chacun des trois (3) groupes linguistiques. Il faut souligner toutefois, que pour chaque mouvement migratoire, la structure par âge et sexe n'est pas différenciée selon la langue.

Le fait de pouvoir entrer séparément dans le modèle chacune des catégories des mouvements migratoires permet de dégager, par le jeu de plusieurs simulations, l'impact possible de chacun de ces mouvements dans la croissance des populations étudiées. C'est ainsi qu'il est possible d'extraire du modèle, pour les besoins spécifiques du ministère de l'Immigration du Québec, l'effet possible d'un changement du volume ou de la composition linguistique des entrées internationales sur la population montréalaise.

Une fois calculés les survivants et le solde migratoire par sexe et année d'âge pour chacun des groupes linguistiques (langue maternelle), il suffit de les additionner pour obtenir la population âgée de plus d'un (1) an, par sexe et âge, au ler juin de l'année t, selon la formule:

$$
\begin{aligned}
& x^{P_{i}}(t)=x-1 P_{i}(t-1) \cdot x-1{ }^{S}(t-1)+x^{M}(t)
\end{aligned}
$$

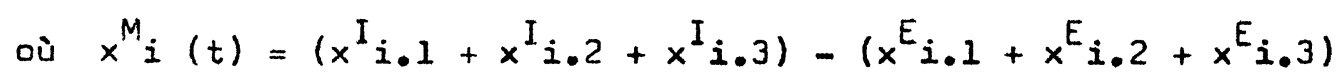


C) Calcul des moins d'un (1) an

Cette équation générale s'applique de façon immédiate à tout âge, c'est-à-dire quel que soit $x$, sauf pour $x=0$. En effet, pour prévoir l'effectif de la population de langue maternelle $i$, de moins d'un (1) an au premier juin de l'année $t$, il faut d'abord prévoir le nombre de naissances $(\mathrm{Ni}$ ) survenues entre les premiers juins des années $t-1$ et $t$.

C.1 Calcul des naissances

Deux (2) postulats ont servi de base aux calculs des naissances.

1) La langue d'usage de la mére détermine la langue maternelle de sa descendance.

En effet, dans le cas où la langue d'usage d'une femme est différente de sa langue maternelle, il est fort probable que cette descendance aura comme langue maternelle, la langue qu'elle parle le plus souvent à la maison.

Ce phénomène, dont on ne peut tenir compte que depuis 1971 , puisque la langue d'usage a étí demandée pour la première fois lors de recensement de 1971, donne à notre modèle un caractère original, puisqu'à no- 
tre connaissances aucune projection publiée jusqu'ici n'a tenu compte de l'effet de cette mabilité linguistique sur les générations ${ }^{1}$. Ce phénomène constitue la mabilité linguistique inter-génération.

2) Les femmes conservent, face à la fécondité, le comportement de leur groupe linguistique d'origine (langue maternelle). Nous avons dû faire cette hypothèse, faute de données sur la fécondité des femmes selon leur langue d'usage. Une telle hypothèse suppose que le transfert linguistique d'une femme n'influence pas son comportement face à la fécondité. Il nous paraît plausible en effet qu'une femme de langue maternelle italienne mais de langue d'usage anglaise ait une fécondité plus proche du groupe italien que du groupe anglais, sans toutefois être exactement identique.

Une fois ces deux (2) postulats posés, nous calculons les naissances de la façon suivante:

1 MM H. CHARBONNEAU et R. MAHEU, dans le cadre des études de la Commission GENDRON, ont tenu compte de la mobilité linguistique inter-génération à partir des concepts d'origine ethnique et de langue maternelle, ne disposant pas a l'époque de connées sur la langue d'usage. 
C.2 Calcul des naissances: langue maternelle française ${ }^{1}$

$N_{1}(t-1, t)=\left[\sum_{x=16}^{45} x-1 V_{1}(t-1) \cdot\left(\frac{\left.\left.x-1 F_{1}(t-1) \cdot x-1 C_{1}(t-1)+x^{F_{1}(t)} \cdot x^{C_{1}(t)}\right)\right]}{2}\right]\right.$

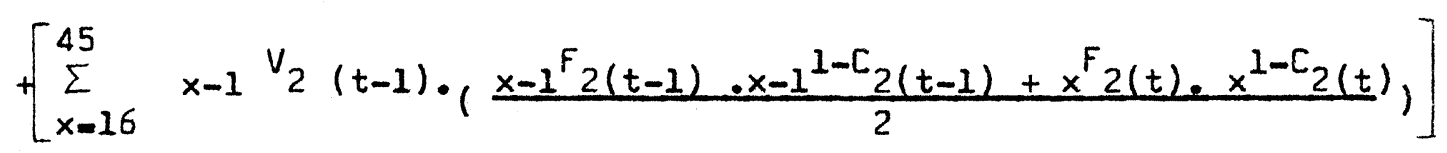

$+\left[\sum_{x=16}^{45} x-1 V_{3}(t-1) \cdot\left(\frac{x-1 F_{3}(t-1), x-1{ }^{C} 3,1(t-1)+x^{F} 3(t), x^{C_{3,1}(t)}}{2}\right]\right.$

où

- $x-1 V_{1}(t-1)=1 e$ taux de fécondité de la population féminine de langue maternelle française d'âge $x-l$, au ler juin de l'année $t-l$.

- $x-1 F_{1(t-1)} \cdot x-1 C_{1}(t-1)=1$ 'effectif de la population féminine

d'âge $x-1$, de langue maternelle française, qui a conservé le français com-

me langue d'usage au ler juin de l'année t-1; $F_{1}$ représentant la popula-

1 Afin de simplifier les formules du modelle, nous avons caractérisé les populations linguistiques de la façon suivante: les variables de la population française ont l'indice 1 , celle de langue maternelle anglaise 2 , et celle des autres langues maternelles 3 . 
tion féminine de langue maternelle française et ${ } l$, la proportion de celle-ci qui a conservé le français comme langue d'usage.

$$
\text { - } x^{F} l(t) \cdot x^{C_{l}}(t)=l \text { 'effectif de la population féminine d'âge } x
$$

de langue maternelle française qui a conservé le français comme langue d'usage, au ler juin de l'année t.

- $x-1 V_{2}(t-1)$ - le taux de fécondité de la population féminine de langue maternelle anglaise d'áge $x-1$, au ler juin de l'année $t-1$.

- $x-1 F_{2}(t-1) \cdot x-11^{1-C_{2}}(t-1)=1$ 'effectif de la population féminine d'âge $x-1$, de langue maternelle anglaise et de langue d'usage francaise, au ler juin de l'année $t-1 ; F_{2}$ représentant la population féminine de langue maternelle anglaise et $\left(1-C_{2}\right)$ étant la proportion de celle-ci ayant adopté le français comme langue d'usage.

- $x^{F}(t) \cdot x^{l-C_{2}}(t)=$ l'effectif de la population féminine d'âge $x$, de langue maternelle anglaise et de langue d'usage française, au ler juin de I'année t. 
- $x-1 V_{3}(t-1)=1 e$ taux de fécondité de la population féminine de langue maternelle "autre", d'âge $x-1$ au ler juin de l'année t-l.

- $x-1 F_{3}(t-1) \cdot x-1 C_{3} \cdot 1 \cdot(t-1)=1$ 'effectif de la population féminine d'âge $x-1$ de langue maternelle "autre" et de langue d'usage française, au ler juin de l'année $t-1 ; F_{3}$ représentant la population féminine de langue maternelle autre et $C_{3.1}$, la proportion de celle-ci ayant adopté le français comme langue d'usage.

$$
\text { - } x^{F_{3}(t)} \cdot x^{C_{3} .1}(t)=1 \text { 'effectif de la population féminine d'âge }
$$
$x$, de langue maternelle "autre" et de langue d'usage française, au ler juin de l'année t.

C.3 Calcul des naissances: langue maternelle anglaise Les calculs pour obtenir les naissances de langue maternelle anglaise sont similaires à ceux que nous venons d'exposer pour les naissances de langue maternelle française.

En effet, les naissances de langue maternelle anglaise d'une (1) année donnée $\mathrm{N}_{2}(t-1, t)$ s'obtiennent en appliquant tout d'abord les taux 
de fécondité des femmes de langue maternelle anglaise $\left(V_{2}\right)$ à l'effectif moyen des femmes de langue maternelle anglaise $\left(F_{2}\right)$ et de langue d'usage anglaise $\left(C_{2}\right)$ de l'année, puis les taux de fécondité des femmes de langue maternelle française $\left(V_{1}\right)$ a l'effectif moyen des femmes de langue maternelle française $\left(F_{1}\right)$ mais de langue d'usage anglaise $\left(1-C_{1}\right)$ enfin les taux de fécondité des femmes de langue maternelle "autre" $\left(V_{3}\right)$ a l'effectif moyen des femmes de langue maternelle autre $\left(F_{3}\right)$ mais de langue d'usage anglaise $\left(C_{3.2}\right)$, selon la formule:

$$
\begin{aligned}
& \left.N_{2}(t-1), t\right)=\left[\sum_{x=16}^{46} x-1 V_{2}(t-1) \cdot\left(\frac{x-1{ }^{F} 2(t-1) \cdot x-1{ }^{C} 2(t-1)+x^{F} 2(t) \cdot x^{C} 2(t)}{2}\right)\right] \\
& +\left[\sum_{x=16}^{46} x-1 V_{1}(t-1) \cdot \frac{\left(x-1{ }^{F}(t-1) \cdot x-1\left(1-C_{1}\right) t-1+x_{1} F_{1}(t) \cdot x\left(1-C_{1}\right) t\right.}{2}\right] \\
& \left.+\left[\sum_{x-16}^{46} x-1 V_{3}(t-1) \cdot \frac{x-1 F_{3}(t-1) \cdot x-1\left(C_{3}, 2\right) t-1+x^{F} 3(t) \cdot x\left(C_{3}, 2\right) t}{2}\right)\right]
\end{aligned}
$$

C.4 Calcul des naissances: langue maternelle autre que française et anglaise

Les naissances de la population de langue maternelle "autre" sont calculées uniquement à partir des femmes de langue maternelle et de langue d'usage "autre". Ceci découle de notre decision d'empêcher les populations de langue maternelle française et anglaise d'effectuer un trans- 
fert linguistique vers une langue d'usage autre que le français et l'anglais. Les naissances pour le tiers groupe se calculent donc d'après la formule abrégée suivante:

$N_{3}=\sum_{x=16}^{46} x-1 V_{3}(t-1) \cdot \frac{x-1 F_{3}(t-1) \cdot x-1{ }^{C} 3(t-1)+x^{F}(t) \cdot x^{C_{3}(t)}}{2}$

- $x-1 V_{3}(t-1)=1 e$ taux de fécondité de la population féminine de langue maternelle "autre", d'âge $x-1$ au ler juin de l'année t-1.

$$
\text { - } x-1 F_{3}(t-1) \cdot x-1 C_{3}(t-1)=1 \text { 'effectif de la population fémi- }
$$

nine d'âge $x-1$, de langue maternelle et de langue d'usage "autre", au ler juin de l'année t-1.

- $x^{F_{3}}(t) \cdot x^{C_{3}}(t)=1$ 'effectif de la population féminine d'âge $x$, de langue maternelle et de langue d'usage "autre" au ler juin de l'année $t$.

C.5 Répartition des naissances par sexe

Le nombre prévu des naissances doit être ensuite réparti selon le sexe. Nous avons adopté un pourcentage de naissances masculines égal 
a $51,4 \%$. Ce pourcentage correspond à la norme généralement admise en

1 a matiere.

C.6 Population totale des moins d'un (1) an par langue maternelle

Pour obtenir le nombre des enfants de moins d'un (1) an au temps

$t$ par sexe et langue maternelle, il suffit d'appliquer aux naissances

féminines et masculines une probabilité de survie entre la naissance et

le premier anniversaire et un solde migratoire.

D) Population totale

La population totale selon le sexe, l'âge et la langue maternelle au ler juin de l'année t s'obtient en ajoutant aux effectifs de moins d'un (1) an les effectifs de plus d'un (I) an abtenus aux étapes précédentes.

\section{$2.5 \frac{\text { Evolution de la population de langue d'usage française, anglaise }}{\text { et autre }}$}

Une fois connues les populations de langue maternelle française, anglaise et autre au temps $t$, il est possible de calculer les populations de langue d'usage au temps $t$ en appliquant aux populations de langue maternelle des taux de conservation ou de perte de la langue maternelle du

1 Les populations selon la langue d'usage ont été caractérisées de la façon suivante: $P_{4}$ concerne la population francophone, $P_{5}$ anglophone et $P_{6}$ allophone. 
moment.

Le taux de conservation à l'âge $x$ et au temps $t$ d'une langue maternelle donnée se définit comme étant la proportion de cette population d'âge $x$ qui a conservé sa langue maternelle comme langue d'usage au ler juin de l'année t.

Les taux de transferts linguistiques étant leur complément, ils représentent les proportions de la population ayant adopté une langue d'usage autre que leur langue maternelle.

Nous obtencns la population de langue d'usage française d'âge $x$ au temps $t$ selon la formule suivante:

$x^{P_{4}}(t)=\left[x^{P_{1}}(t) \cdot x^{C_{1}}(t)\right]+\left[x^{P_{2}}(t) \cdot x^{1-C_{2}}(t)\right]+\left[x^{P_{3}}(t) \cdot x^{C_{3}} \cdot 1(t)\right]$

๑ù

- $x^{P} 4(t)$ - 1'effectif de la population de langue d'usage française d'âge $x$ au temps $t$.

- $x^{P} l(t) .=1$ leffectif de la population de langue maternelle française d'âge $x$ au ler juin de l'année t. 
- $x^{C_{1}}(t)=$ le taux de conservation du français comme langue d'usage a l'âge $x$, au ler juin de l'année t.

- $x^{P} 2(t)=1$ effectif de la population de langue maternelle anglaise d'âge $x$ au ler juin de l'année t.

- $x^{1-C_{2}}(t)=$ le taux de transfert de l'anglais au français à l'âge $x$, au ler juin de l'année $t$.

- $x^{P_{3}}(t)=$ l'effectif de la population de langue maternelle autre que française et anglaise d'âge $x$ au ler juin de l'année t. - $x^{C_{3.1}}(t)=$ le taux de transfert de la population de langue maternelle "autre" au français à l'âge $x$, au ler juin de l'année t.

On calcule la population de langue d'usage anglaise $\left(P_{5}\right)$ selon les mêmes principes utilisés pour la population de langue d'usage française.

L'équation mathématique prend la forme suivante en ce qui concerne le calcul des effectifs de la population de lanque d'usage anglaise d'âge $x$ au temps $t$ :

$x^{P_{5}}(t)=\left[x^{P_{2}}(t) \cdot x^{C_{2}}(t)\right]+\left[x^{P_{1}}(t) \cdot x^{1-C_{1}}(t)\right]+\left[x^{P_{3}}(t) \cdot x^{C_{3}} \cdot 2(t)\right]$ Quant à la population de langue d'usage "autre" $\left(P_{6}\right)$, son calcul 
se limite à l'équation suivante:

- $x^{P_{6}}(t)=x^{P_{3}}(t) \cdot x^{C_{3}}(t)$

\section{3- CONCLUSION}

Tous les facteurs de la croissance des populations linguistiques ont été distingués dans le modêle. C'est ainsi, par exemple, qu'il retient non pas des soldes migratoires pour chaque groupe linguistique mais les entrées et les sorties des différents types de migration. Ceci le rend particulièrement souple et permet d'étendre son utilisation à d'autres fins que l'btude actuelle. Ce modele permet en effet de mesurer par le jeu de plusieurs simulations les effets d'ici 2001 d'un changement d'un des facteurs de croissance des ou d'une seule des populations linguistiques non seulement sur la composition linguistique future de la population montréalaise mais également sur son volume, son taux de croissance et sa structure par âge. 